\section{§21. The Radial Electric Field in a Tokamak with Reversed Magnetic Shear}

Zhu, P., Horton, W. (Inst. Fusion Studies, Univ. Texas), Sugama, $\mathrm{H}$.

Neoclassical theory with the impurity rotational velocity is used to evaluate the radial electric field, $E_{r}$, in a tokamak [1]. Two transport measures of the effect of the $E_{r}$ shear are compared for the reversed shear (RS) and enhanced reversed shear (ERS) discharges in Tokamak Fusion Test Reactor (TFTR) [2]. It is shown that the combined $E_{r}$ and magnetic shear measure, $\Upsilon_{s}$, from linear stability theory gives a higher correlation with the observed transition between the two discharges than the vorticity measure $\omega_{s}$ from $E_{T}$ shear alone.

Figure 1 shows the radial electric field $E_{r}$. calculated for the RS and ERS discharges. In both discharges, $E_{r}$ radial profile has a 'well' structure inside the central region where the safety factor $q$ is minimum. As time evolves, $E_{r}$ 'well' develops from a rather shallow 'well' to much deeper one in both the RS and the ERS discharges. The $E_{r}$ in the ERS discharge is significantly larger and steeper than that in the $R S$ discharge at all the time stages.
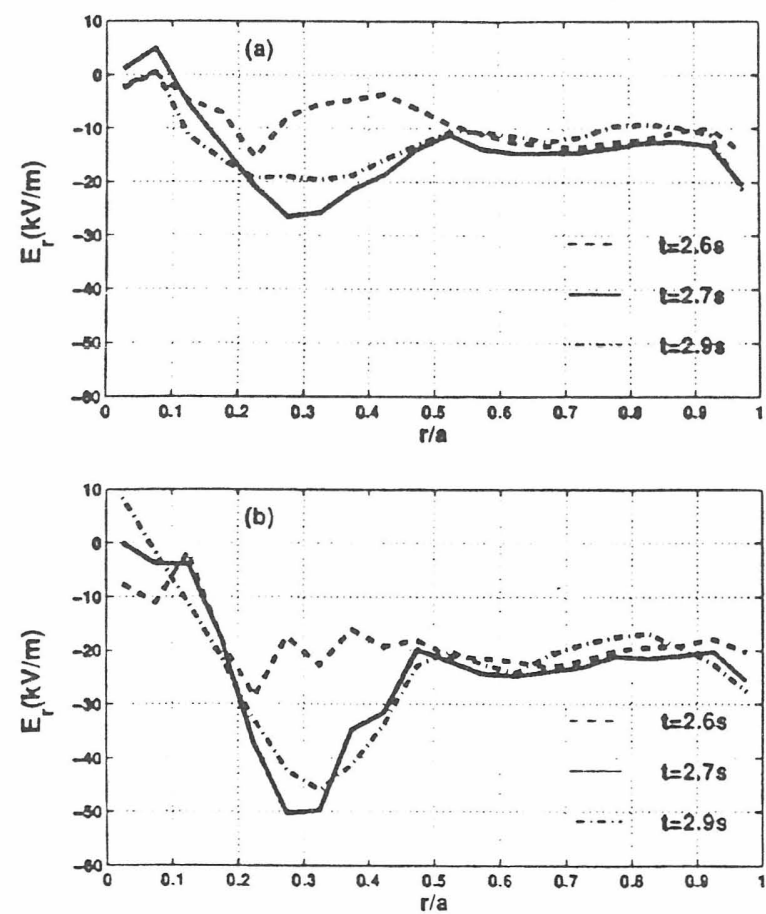

Fig.1. The time evolution of $E_{r}(r, t)$ for (a) the RS discharge and for (b) the ERS discharge at times before $(t=2.6 \mathrm{~s})$ and after $(t=2.7 \mathrm{~s}, 2.9 \mathrm{~s})$ the bifurcation.
The Hahm-Burrell $\mathbf{E} \times \mathbf{B}$ flow shearing rate $\omega_{s}$ is given by

$$
\omega_{s}=\frac{\Delta \psi_{0}}{\Delta \phi_{0}} \frac{\partial^{2} \Phi_{0}(\psi)}{\partial \psi^{2}} \simeq\left|\frac{R B_{\theta}}{B_{\phi}} \frac{\partial}{\partial r}\left(\frac{E_{r}}{R B_{\theta}}\right)\right|
$$

where $\Delta \%_{0}$ and $\Delta \phi_{0}$ are the ambient radial and toroidal correlation lengths measured in units of poloidal flux and radians respectively [3]. The other relevant measure of $E_{r}$ shearing rate is the linear stability theory parameter

$\Upsilon_{s}=\frac{\text { flow shear }}{\text { magnetic shear }}=\frac{L_{V_{E}}^{-1}}{L_{s}^{-1}} \simeq \sqrt{\frac{m_{i}}{T_{\epsilon}}}\left|\frac{R \partial_{\psi}\left(E_{r} / R B_{\theta}\right)}{\partial_{\psi} \ln q}\right|$

which measures the stabilizing effects of $\mathrm{E} \times \mathrm{B}$ flow shear in sheared magnetic fields [4]. Figure 2 shows these two measures in the RS and ERS discharges.
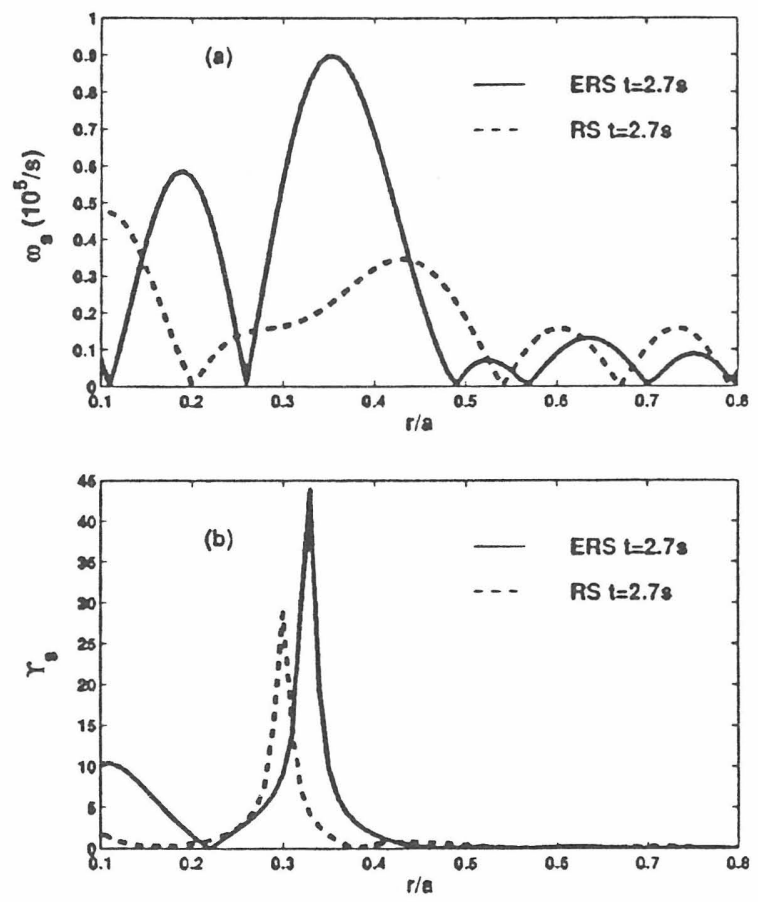

Fig.2 T(a) Comparison of the Hahm-Burrell shear rate in the RS and ERS discharge at $t=2.7 \mathrm{~s}$. (b) Comparison of the linear stability measure $\Upsilon_{s}$ for the RS and ERS discharges.

References

1) Zhu, P., Horton, W., and Sugama, H. : Phys. Plasmas 6 (1999) 2503.

2) Mazzucato, E., al. : Phys. Rev. Lett. 77 (1996) 3145.

3) Hahm, T. S., and Burrell, K. H. : Phys. Plasmas 2 (1995) 1648.

4) Hamaguchi, S., and Horton, W. : Phys. Fluids B 4 (1992) 319. 